\title{
PHENIX Beam Energy Scan Results
}

\author{
R.A. Soltz (for the PHENIX Collaboration) ${ }^{1}$ \\ Physics Division, Lawrence Livermore National Laboratory, Livermore, CA 94550, USA
}

\begin{abstract}
We present results from the PHENIX Experiment for Au+Au collisions with $\sqrt{s_{N N}}=7.9,19.6,27,39,62$, and 200 GeV. Measurements of the charged particle multiplicity at central rapidity scale linearly with the number of participant quarks for $\sqrt{s_{N N}}=62 \mathrm{GeV}$ and above; for $\sqrt{s_{N N}}=27 \mathrm{GeV}$ and below the multiplicity scales with the number of participant nucleons. For the HBT radii we perform a linear interpolation for radii from PHENIX, STAR, and ALICE to a $m_{T}=0.26 \mathrm{GeV}$ and calculate ratios and differences in quadrature at this value of the transverse mass. We observe a non-monotonic behavior near $\sqrt{s_{N N}}=19 \mathrm{GeV}$ in the form of a peak in $R_{o}^{2}-R_{s}^{2}$ and a dip in $\left(R_{s}-\sqrt{2} \bar{R} / R_{l}\right.$.
\end{abstract}

Keywords: Multiplicity, Femtoscopy, Quark-gluon plasma

\section{Introduction}

The combination of a cross-over transition at $T=154 \pm 9 \mathrm{MeV}$ at zero baryon density calculated in Lattice QCD [1] and a first order phase transition at finite density suggested by chiral effective models implies the existence of a critical point in the QCD phase diagram [2]. The ongoing Beam Energy Scans (BES) at RHIC are designed to explore regions of high baryon density with the goal of identifying signatures of the critical point. RHIC has generated $\mathrm{Au}+\mathrm{Au}$ collisions at $\sqrt{s_{N N}}$ of 7.7, 11.5, 19.6, 27, 39, and $62 \mathrm{GeV}$. Measurements of baryon and charge fluctuations are expected to serve as the primary signatures of critical behavior, however, it is instructive to examine a full set of experimental signatures in order to fully explore the evolution and freeze-out of heavy ion collisions as a function temperature and baryon density. In this paper we present recent results on multiplicity scaling and HBT radii and their dependence on Au+Au collision energy as measured by the PHENIX Experiment.

\section{Multiplicity Scaling}

Fig. 1 shows charged particle multiplicity measurements by PHENIX for various collision energies as a function of collision centrality. The left panel shows the scaling by nucleon participants, whereas the right panel shows scaling by quark participants. All data sets in both panels are normalized by the multiplicity measured for the $0-5 \%$ centrality bin, in order to show more clearly the deviations from linear scaling with either nucleon or quark participants. In this form it is evident that the multiplicity at central rapidity follow quark scaling for collision energies of $\sqrt{s_{N N}}$ of $62 \mathrm{GeV}$ and above, but follow nuclear scaling at $27 \mathrm{GeV}$ and below. This variation in the scaling of produced particles is an important consideration when studying signatures of the critical behavior, as the variation in particle production mechanisms may have implications for measurements of fluctuations. For a complete analysis of the multiplicity and transverse energy scaling see [3].

\footnotetext{
${ }^{1} \mathrm{~A}$ list of members of the PHENIX Collaboration and acknowledgements can be found at the end of this issue.
} 

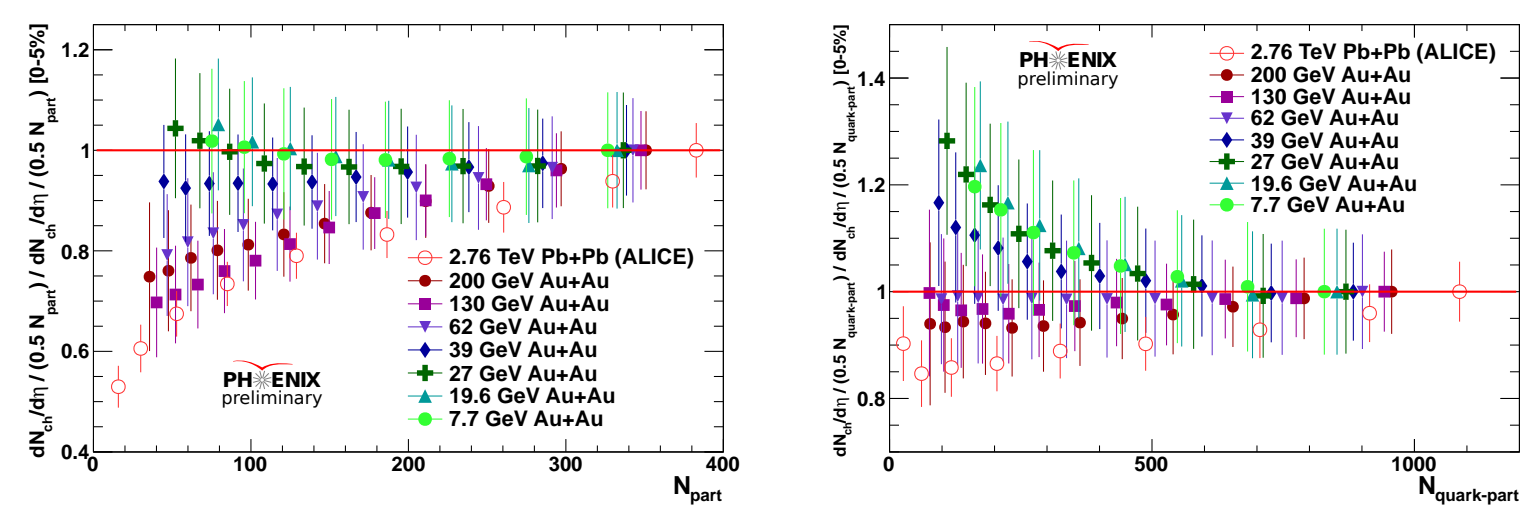

Figure 1. Charged particle multiplicity per participant nucleon/quark at mid-rapidity as a function of centrality expressed as participant nucleon (left) and participant quark (right). All data are normalized to the value in the 0-5\% centrality bin and errors shown are systematic.

\section{HBT Radii Scaling}

PHENIX has also measured HBT radii for pion pairs in Au+Au collisions at $\sqrt{s_{N N}}=200,62$, and $39 \mathrm{GeV}$. The correlation functions were calculated in the longitudinal co-moving system and fit in the Bersch-Pratt coordinate system, where $R_{\text {long }}$ is parallel to the beam direction, $R_{\text {out }}$ is parallel to the transverse component of the pion pair momenta, and $R_{\text {side }}$ is along the remaining orthogonal direction. Coulomb corrections were performed using the corehalo correction, as described in [4], which also provides more details on tracking, particle identification, and two-track selection.

In Fig. 2 we show that HBT radii for 39, 62, and $200 \mathrm{GeV}$ are well described by the empirical formula, $R_{i}=$ $a+b / \sqrt{m_{T}}$. This differs from the more traditional formulas motivated by longitudinally boost invariant hydrodynamic expansion in the transverse direction, however, the $\chi^{2}$ values for the empirical formula are just as good as the two parameter fits suggested in [5, 6] and previously used by PHENIX in [7]. In Fig. 3 we show that the radii for $\sqrt{s_{N N}}=200 \mathrm{GeV}$ for $\mathrm{Au}+\mathrm{Au}$ and $\mathrm{Cu}+\mathrm{Cu}$ scale linearly in $\bar{R}=\sqrt{\frac{1}{\sigma_{x}^{2}}+\frac{1}{\sigma_{y}^{2}}}$, the harmonic mean of the initial rms overlap regions in $x$ and $y$, as calculated using a Monte Carlo Glauber model. This scaling parameter appears to capture the dynamic expansion which govern the final state freeze-out lengths of homogeneity.

In the left panel of Fig. 4 we show the difference in quadrature for $R_{\text {out }}$ and $R_{\text {side }}$ as a function of center-ofmass energy for $0-5 \%$ centrality. In the absence of dynamical correlations this quantity is equal to the emission duration divided by the mean transverse pair velocity. Although the presence of complex dynamics complicatpes the interpretation, the non-monotonic behavior may indicate an increase in the emission duration in the vicinity of the peak at $\sqrt{s_{N N}}=19 \mathrm{GeV}$. This could be due to an extended re-scattering phase for this incident beam energy, as well as a softening of the equation of state near the deconfinement transition.

In the right panel of Fig. 4 we plot the ratio of $R_{s}-\sqrt{2} \bar{R}$ to $R_{l}$. The numerator is indicative of the expansion from initial state to final transverse length of homogeneity, and the denominator is indicative of the total collisions duration from initial overlap of the nuclei until freeze-out. Thus the minimum is also qualitatively consistent with a softening in the equation of state for this beam energy.

\section{Conclusions}

In summary, measurements of the charged particle multiplicity at mid-rapidity and HBT radii as a function of incident beam energy are consistent with a change in dynamics in the range $\sqrt{s_{N N}}=19-27 \mathrm{GeV}$. The mid-rapidity multiplicity measurements indicate a shift from quark scaling to nucleon dominated particle production dynamics with decreasing center-of-mass energy. We observe a peak in the quantity, $\left(R_{o}^{2}-R_{s}^{2}\right)$ and a dip in $\left(R_{s}-\sqrt{2} \bar{R}\right) / R_{l}$. The former is consistent with an extension of the emission duration that would accompany a prolonged re-scattering phase, 


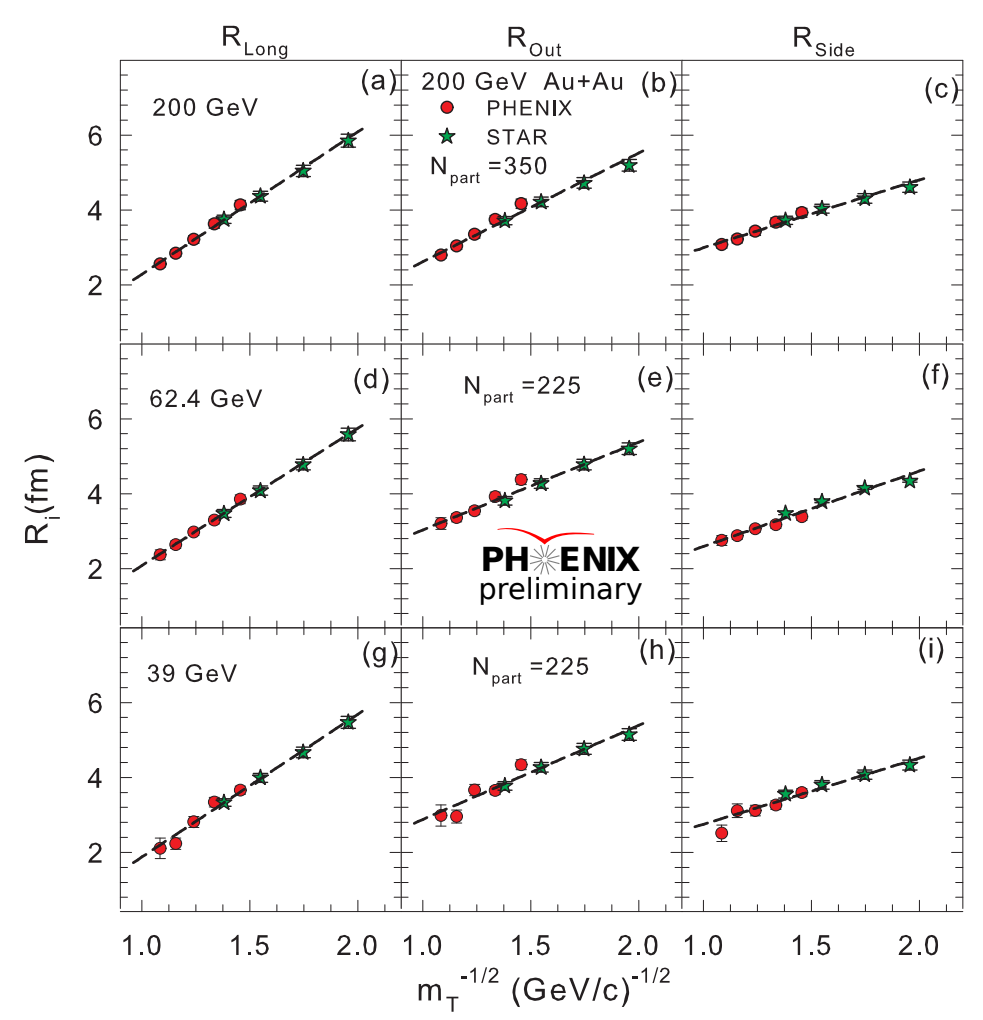

Figure 2. HBT radii, $R_{\text {long }}$ (left), $R_{\text {out }}$ (center), and $R_{\text {side }}$ (right) vs. $1 / \sqrt{m_{T}}$ for PHENIX and STAR for Au+Au collisions with $\sqrt{s_{N N}}=200 \mathrm{GeV}$ (top), $64 \mathrm{GeV}$ (center), and $39 \mathrm{GeV}$ (bottom).

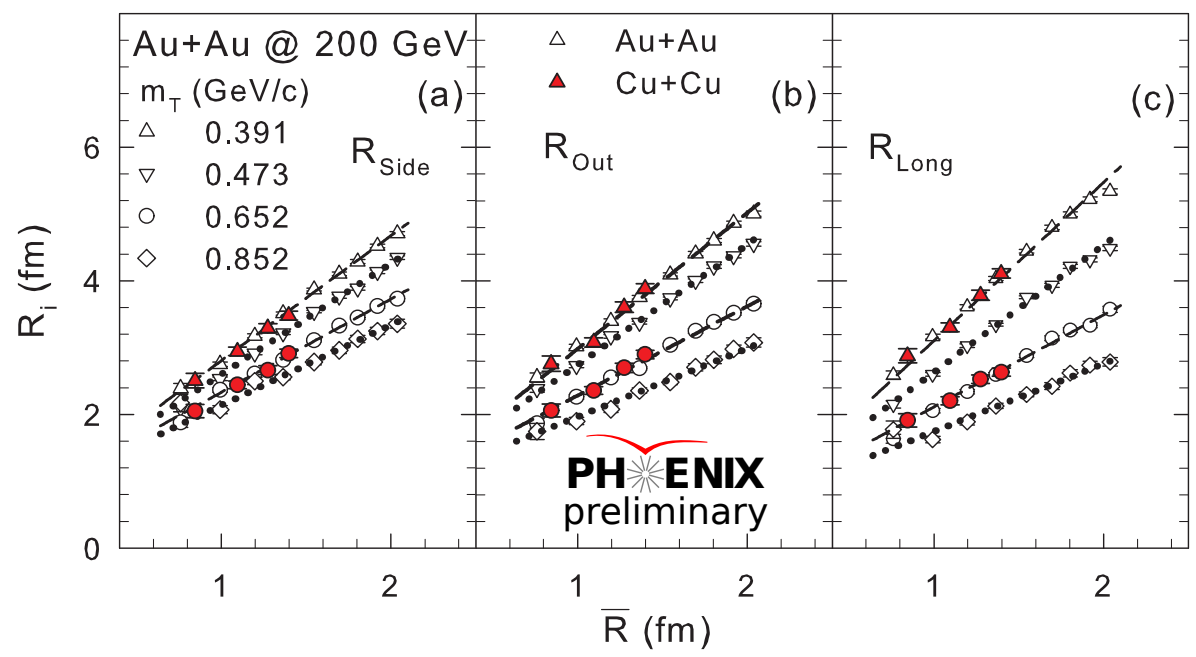

Figure 3. $R_{\text {side }}$ (left), $R_{\text {out }}$ (center), and $R_{\text {long }}$ measured by PHENIX as a function of $\bar{R}$ for $\sqrt{s_{N N}}=200 \mathrm{GeV} \mathrm{Au}+\mathrm{Au}$ and $\mathrm{Cu}+\mathrm{Cu}$ collisions. The dashed and dotted lines are linear fits to the radii for different values of $m_{T}$. 


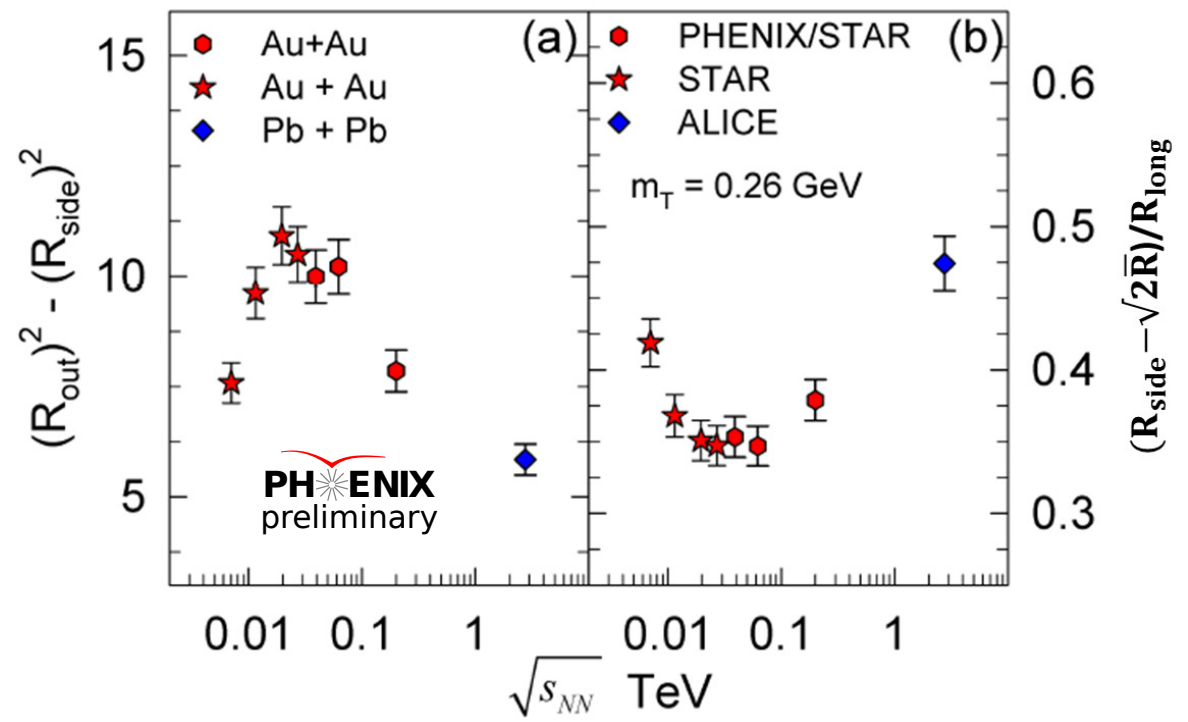

Figure 4. $R_{o}^{2}-R_{s}^{2}$ (left) and $\left(R_{s}-\sqrt{2} \bar{R}\right) / R_{l}$ (right) as a function of $\sqrt{s_{N N}}$. Data from STAR (red stars) [8] and PHENIX and STAR combined (red circles) are interpolated to $m_{T}=0.26 \mathrm{GeV}$ from linear fits to $R_{i}=a+b / \sqrt{m_{T}}$. The radii from ALICE (blue diamonds) [9] were extrapolated to the same $m_{T}$ value from radii measured in $\sqrt{s_{N N}}=2.76 \mathrm{TeV}$.

and both are consistent with a large fraction of the collision evolution passing though the softest point the equation of state. Detailed models employing both hydrodynamic and microscopic transport are needed before any quantitative conclusions can be drawn from these results.

This material is based upon work supported by the U.S. Department of Energy, Office of Science, Office of Nuclear Physics under contract number DE-AC52-07NA27344.

\section{References}

[1] A. Bazavov, et al. [HotQCD Collaboratin], Phys. Rev. D 85, 054503, 2012.

[2] M. Stephanov, K. Rajagopal, and E. Shuryak, Phys. Rev. Lett. 81, 4816, 1998.

[3] S. Adler, et al. [PHENIX Collaboration], Phys. Rev. C 89, 044905, 2014.

[4] A. Adare, et al. [PHENIX Collaboration], arxiv:1404.5291, 2014.

[5] A. Makhlin and Y. Sinyukov, Z. Phys. C 39, 69, 1988.

[6] S. Chapman, P. Scotto, P., and U. Heinz, Phys. Rev. Lett, 74, 4400, 1995.

[7] K. Adcox, et al. [PHENIX Collaboration], Phys. Rev. Lett. 88, 192302, 2003

[8] L. Adamczyk, et al. [STAR Collaboration], arxiv:1403.4972, 2014.

[9] K. Aamodt, et al. [ALICE Collaboration], Phys. Lett. B 696, 328, 2011. 